\title{
Eye.Breathe.Music: creating music through minimal movement
}

\author{
Sam Bailey, Adam Scott, Harry Wright, lan Symonds, and Kia Ng \\ ICSRiM - University of Leeds, \\ School of Computing, School of Electronic and Electrical Engineering, and School of Music, \\ Leeds LS2 9JT, UK \\ ebm@icsrim.org.uk www.icsrim.org.uk
}

\begin{abstract}
Eye.Breathe.Music is a musical interface that can be controlled by movement of the pupils and breath pressure. The interface uses a pressure sensor to measure breath intensity; a camera to track eye movement and a display to provide the user with interactive feedback. This paper discusses the background research into the instrument, how this was used to design and construct the system, an evaluation of the resulted prototype, future development and possible applications.
\end{abstract}

Eye. Tracking. Breath. Sensor. Controller. Instrument. Movement. Multimedia. Interactive.

\section{INTRODUCTION}

Conventional musical instruments are usually played through observable physical gestures such as hand movements. The aim of Eye.Breathe.Music is to create a musical interface using comparatively smaller and subtle motions to similar effect. The instrument, named the Lumiselo, is played through eye movement and breathing, making the movements extremely subtle with minimal space requirements.

The instrument could also act as a controller for existing sounds. A new sound, unique to the instrument has been created to give the instrument an audio identity.

As it is effectively an electronic wind instrument, users will learn to plan phrases in a similar way to playing a wind instrument so they do not run out of breath. The user will also have the ability to perform techniques such as flutter tonguing, growling and circular breathing to their advantage.

\section{BACKGROUND RESEARCH}

Kim et al. (2007) describe a system for performing electronic music, where a video-based eye movement recording system is used to control sounds. Different types of tracking are discussed in this paper such as electrophysiological methods, (a method of recording different potentials generated between electrodes placed either side of the corneoretinally polarized eye, also referred to as electro-oculogram, EOG), infrared reflective methods and video recording.

EyesWeb is a research project that explores extending musical language by analysing and processing expressive gestures (Castellano et al. 2007). A camera tracks the user's body movement and creates music. This is typically for larger movement such as dance. This is similar to our project as we want to record gestures to create music.

The Gypsy Motion Capture System (http://www.metamotion.com/) is another motion capture solution. This system does not use cameras, but mechanical arms and legs attached to the users. These then translate the data from the user onto a computer and can be used as musical controller (Rothwell, 2006).

The Pipe (Scavone, 2003) is a musical instrument modelled around the design of a wind instrument. The user blows into a pressure sensor within the pipe and uses their fingers to control force sensing resistors, two switches, two potentiometers and an accelerometer. The Pipe uses static airflow as opposed to a dynamic free-flowing system traditionally used in wind instruments and current MIDI wind controllers.

Silva et al. (2004) describe a system that tracks movements of the lower face and use movements of the mouth to control audio synthesis such as a 'wah-wah' effect on a guitar. 
EyeMusic (Hornof \& Sato, 2004) is a system that tracks eye movement for electronic music compositions. In this paper the tracked pupils can control the music or the music can respond to the movement of the pupils. This takes two users, a composer and an eye performer. The eye performer uses a chin rest to keep his/her head still and a camera mounted near the computer tracks where they are looking.

Several commercial and research systems use the head mounted approach to give the user freedom such as the Eyewriter (http://www.eyewriter.org/) and openEyes (Babcock, 2006). Both these systems use a camera placed on thick wire and then attached to glasses. This allows the camera to be moved for each individual user.

\section{DESIGN AND DEVELOPMENT}

The eye controls the pitch that the instrument sounds. The user's breath determines and shapes the dynamics of the note to offer expressive controls such as phrasing, volume profiles and different articulation techniques.

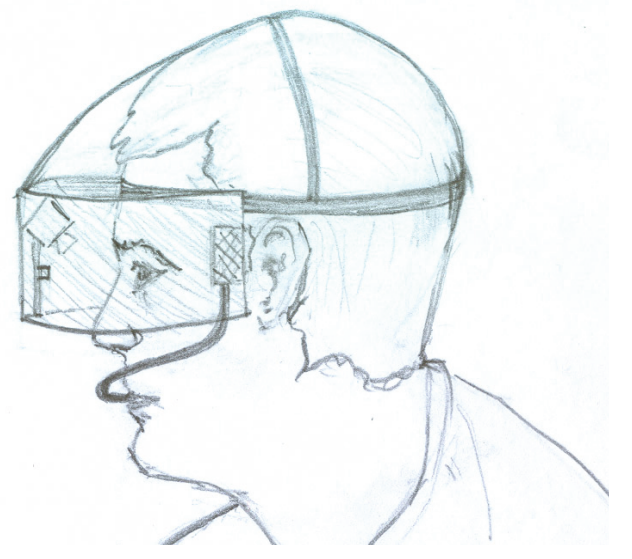

Figure 1: Conceptual design drawing

In order for breath intensity to control the volume of the note, a pressure sensor is used to measure the intensity of the breath and outputs a voltage value accordingly. These values are digitised, sent to the computer and the data is conditioned and analysed to provide expressive controls. Figure 1 illustrates the conceptual design of the interface.

Lumiselo uses a camera mounted on goggles rather than a fixed camera and chin rest. Not only is this beneficial for portability, enabling the user to move their head whilst playing, but it also makes it easier for the computer to track the pupil. An infrared LED with a safe level of intensity is directed into the eye to create reflections. The use of infrared light source reduces the spill of visible light from the room and improves tracking results. The pupil appears as a well-defined circle, due to the light entering the eye and reflecting off the retina.

A set of LEDs are aligned into a grid for the display, which is mounted on a visor. On the LED-grid display there are three rows of twelve bi-colour LEDs, this allows the chromatic scale in three octaves. When the user looks at an LED the camera tracks the eye, the coordinates of the pupil are then found using Max/MSP/Jitter and the correct pitch to sound at the relevant volume.

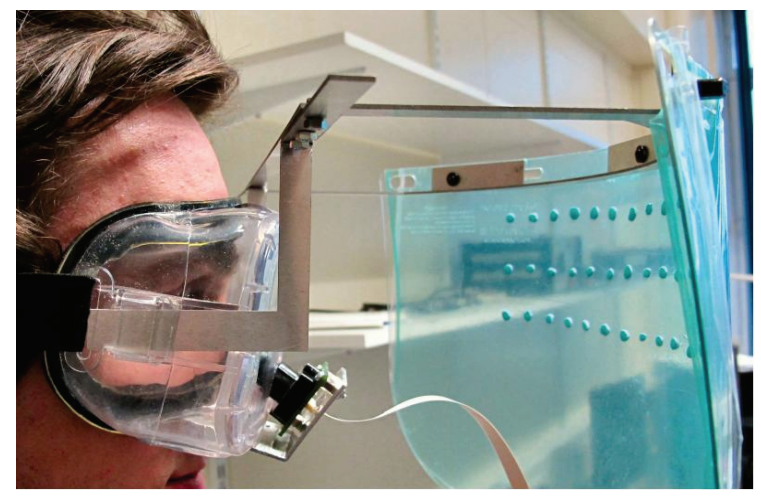

Figure 2: A photo of the first prototype

The bi-colour LED that the user is looking at changes colour and varies in brightness according to the intensity of the user's breathing. This makes the display interactive and gives the user feedback as to which note they are sounding and how loud it is being played. An overview of the system is represented in Figure 3.

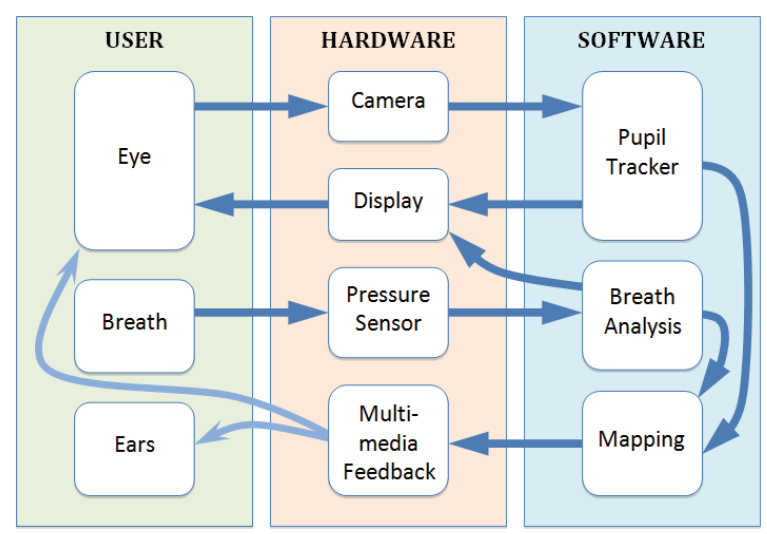

Figure 3: Overview of the system architecture

The intensity of the user's breath is shown through the brightness of the LEDs. This will be done through multiple Op-amps giving each LED different voltage levels separately in time (using time division multiple access).

The headset makes the instrument compact and appealing. It also allows head movement to have 
no effect on the location of the user's pupil in respect to both the camera and display.

Ideally it will be possible for the camera to be fixed over either eye, allowing the user to choose. Although, it is essential for the camera to be in a completely fixed position while the instrument is being played and this must not be compromised. Similarly, the LED display (situated directly in front of the user's eyes, $17 \mathrm{~cm}$ from the eyes to allow a minimal focus range) will need to have two configuration settings, depending on which eye is used.

Changing the lighting in front of the user's eyes will cause pupils to dilate. This may cause a problem when tracking the pupils; however using multiplexing techniques on the LED-grid will allow there to be the same intensity of brightness at all times. If the user breathes as hard as they can, one LED will be lit on its own, at 'full' brightness, while if the user were to not be breathing at all, all the LEDs would shine at a level of brightness that, when they are all added together, equals that of one LED at 'full' brightness. Therefore the same level of total light will always be emitted by the display.

Research was conducted to discover the way in which the eye moves and focuses on objects. This helped gain understanding of how the average human eye will react to the eye tracking system and LED display. There are two types of eye movement that can be defined as 'external' and 'internal.' Internal movements tend to only move some of the inner parts of the eye, such as the width of the pupil, making them impossible to track. External movements however, shift the whole globe of the eye; these are the movements that the camera will be tracking. When fixated, the eyes still go through low amplitude, high frequency tremors (Wade et al., 2005), these slight movements will need to be accounted for.

Each user will have one eye stronger than the other, due to ocular dominance. That is why the instrument will have to be made so that it can be calibrated for each eye, allowing the user to input their preference.

There are three rows of LEDs, each one being a different octave (see Figure 4). Consequently if the user looks at an LED, and then subsequently looks at the one below, the instrument will sound a note an octave lower. Likewise, if the user looks at an LED directly above the previous, the note will move up an octave.

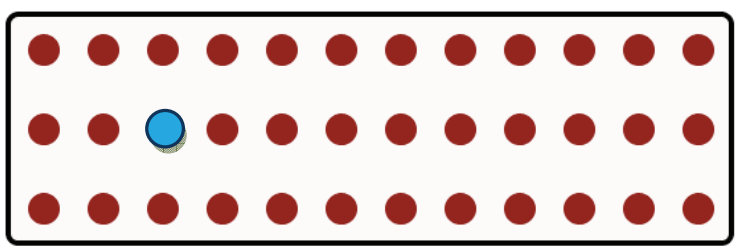

Figure 4: LED layout design

The location of the LEDs used in the display of the first system and the corresponding coordinates relating to pitch can only be decided once the average user's field of view has been researched.

Field of view is determined by the magnitude of the angle a person is able to correctly see at the edge of their visual range. To find how the field of view varies from person to person, a number of different subjects took the test. The subject was asked to close one eye and recordings were taken of how far each side they could focus comfortably. This was recorded at a distance of $10 \mathrm{~cm}, 20 \mathrm{~cm}$ and $30 \mathrm{~cm}$ away from a wall. The test was then repeated using the other eye.

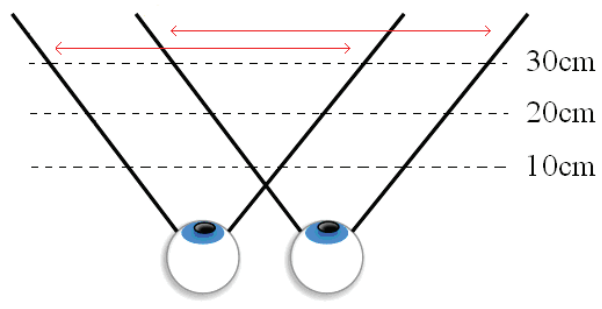

Figure 5: A diagram indicating the field of view for each eye and the different distances used for testing

\section{LUMISELO}

Lumiselo is designed to suit the features of the average user. Numerous tests were performed to find out how the average user will interact with each part of the instrument.

\subsection{The breath sensor}

Out of the numerous pressure sensors tested the Freescale MPXV5010 gave the best results. Atmospheric pressure on the sensor created a voltage output of $0.265 \mathrm{~V}$. However, if this were tested at a different altitude, the result would be different. Therefore the final program must take into account the initial value and set this as 0 .

Using an empty plastic bottle with the pressure sensor inside, measurements were taken. The maximum pressure reached by all subjects was 4.97V. However, creating this pressure was uncomfortable for the user. The comfortable maximum was $3 \mathrm{~V}$. Users found it easy to keep a 
steady pressure within a range of $0.05 \mathrm{~V}$ of the required pressure, this allows for a large range of values.

For the final prototype a tube is used, the pressure sensor is mounted in one end and the other is constantly in the user's mouth. During testing it was found that it was possible to maintain pressure whilst breathing through the nose. Testing proved that not maintaining any pressure in the mouth and breathing through the nose could increase the pressure up to $0.48 \mathrm{~V}$. For this reason, the threshold of the note sounding has been set at $0.5 \mathrm{~V}$.

The static pressure system is more hygienic as it does not require a humid airflow, for this reason it also means there is a decreased chance of electrical components getting wet. As no air is escaping between the contact of the pipe and the users mouth, it is possible to maintain normal breathing through the nose whilst playing, eliminating the need to pause for breath. However, for a wind player, the change from dynamic to static breath pressure could seem unnatural.

Sucking on the pressure sensor was tested as it could possibly be used as another control input. It was possible to create voltages down to $0.035 \mathrm{~V}$ whilst sucking. This would only leave a range of $0.23 \mathrm{~V}$ from the atmospheric pressure, and with steps of $0.05 \mathrm{~V}$ there would only be 4.6 velocity values, thus sucking was deemed redundant.

Using the Microchip PIC16F877 microcontroller the analogue data from the sensor was converted into digital serial. With a FTDI UM232R Serial-to-USB converter and a PicKit 2 the PIC chip was built with a simple ADC code in MPLab and connected to the computer. The serial code was then inputted into Max/MSP using the serial object.

\subsection{Eye movement}

Numerous subjects were recorded reading out the numbers one to twelve written in a line on a piece of A4 paper. This proved whether the movements of the pupils were large enough to measure. The results showed that if the eye moved quickly from far left to far right, a USB camera would not be able to follow it in a continuous stream smoothly. This meant that the camera would need a high bandwidth connection.

\subsection{Eye tracking}

Using the cv.jit.track object in Max/MSP, a program was formed that tracks a given point. Although successful it was not efficient enough as the point being tracked would often move.
An infrared LED has very low levels of emission compared to an IR laser and at these levels is not unhealthy for the human eye. Using cv.jit.centroids, the pupils can therefore be tracked much more effectively. With this information a suitable camera that could read IR was used.

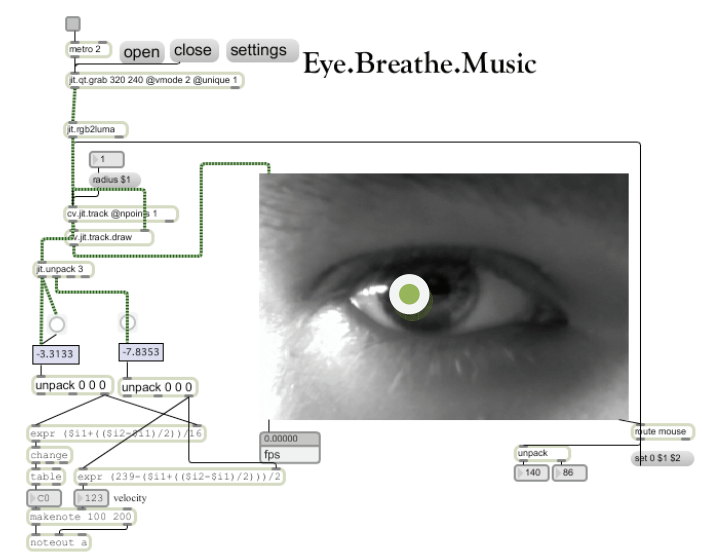

Figure 6: A Max patch using cv.jit.track. The dot on the eye is the object being tracked

To calibrate the system the user must look at the top left and top right LEDs, the program then calculates the positions of the remaining LEDs. The graphical user interface in Max/MSP allows the user to change a wide range of configurations and settings. It is possible to change the instrument, change the key and scale, save and edit preset setting.

\section{CONCLUSION}

Lumiselo integrates the hardware setup and software code as presented above.

The software program, which processes the data, has been developed using Max/MSP/Jitter and allows for two-way communication between the user and the computer. The camera interprets the position of the user pupils; this is communicated to the computer along with the intensity of the user's breath. This information is then transferred from the computer to the LED-grid display.

From the testing and validations carried out so far, we believe Eye.Breathe.Music is a flexible musical interface with an original combination of control inputs and outputs that allow users to express themselves through music with minimal physical movements.

\section{FUTURE WORK}

Currently we are working on the validation of the overall system. Numerous quantitative tests have 
been taken measuring inaccuracies such as the latency of the system. Various designs have been drawn to create a more comfortable and fashionable headset. A group of musicians have been invited to test out the instrument to provide qualitative feedback.

It is hoped that the future development of the first system will work towards the inclusion of a wireless interface. The user will then be free to move without being restricted by cables.

In its current state, some parts of the device, such as selecting a voice for the instrument and opening or closing the patch, must be done with the use of a computer interface (such as a mouse). This will be developed so it is completely hands free.

Although the LED-grid display gives the user a form of interactive feedback, this still does not fully emulate the feeling of playing an acoustic wind instrument. Physical vibration is a huge part playing; this could be produced electronically and triggered using the breath sensor.

In future revisions of the system, it is hoped that the system could be built without the use of a headset by means of a projected display or monitor and a fixed camera. If a computer program could be calibrated to recognise the users face, the program could take into account the position of the users head and from this locate the coordinates of the pupils in reference to the location of the face. The primary function of this system would be identical to the current one; however the user would not be restricted within the confines of the headgear. Carrying on with the current theme of a facially controlled instrument, the velocity of the note could either still be controlled by breath pressure, or by some recognisable variance in facial movements that could be tracked, such as smiling.

In future revisions of the system, the potential for controlling musical systems using the eyes and breathing will be explored further. It could be used to compose, through simply writing notation by operating a cursor with the eyes, or in real time using a system that works like a sequencer. Such a system could even work as a training package, improving the user's ability to control their eyes for use in eye-controlled software.

Visuals could be created from the eye movement that takes place while the instrument is being played. This would provide an audience with something to look at, improving the merit of the instrument being performed live.

Using a system containing multiple cameras, audience members could control of aspects of the performance using their eyes, increasing the level of interactivity and blending the divide between the performer and audience.

We look forward to future work on the Eye.Breathe.Music system, and with succeeding revisions, hope to continue to research and implement further methods of human interaction with music.

\section{REFERENCES}

Babcock, J., Li, D. and Parkhurst, D. (2006) openEyes: a low-cost head-mounted eye-tracking solution. The Human Computer Interaction Program lowa State University, lowa.

Castellano, G., Bresin, R., Camurri, A. and Volpe, G. (2007) Expressive control of music and visual media by full-body movement. In Proceedings of the 7th international conference on New Interfaces for Musical Expression (NIME07), 6-10 June 2007, pp. 390-391. ACM, New York, USA.

\section{Eyewriter. http://www.eyewriter.org/ (24 March} 2010).

Hornof, A. and Sato, L. (2004) EyeMusic: Making Music With The Eyes. In Proceedings of the 2004 International Conference on New Interfaces for Musical Expression (NIME), Hamamatsu, Japan, 35 June 2004, pp. 185-188. National University of Singapore, Singapore.

Kim, J., Schiemer, G. and Narushima, T. (2007) Oculog: Playing with Eye Movements. In Proceedings of the 7th International Conference on New Interfaces for Musical Expression (NIME07). 6-10 June 2007, pp. 50-55. ACM, New York, USA.

Rothwell, N. (2006)

http://www.soundonsound.com/sos/oct06/articles/s onalog.htm (24 March 2010)

Scavone, G. (2003) The Pipe: Explorations with Breath Control. In Proceedings of the 2003 conference on New interfaces for musical expression. Montreal, Canada, 22-24 May, pp. 1518. National University of Singapore, Singapore.

Silva, G., Smyth, T. and Lyons, M. (2004) A Novel Face-tracking Mouth Controller and its Application to Interacting with Bioacoustic Models. In Proceedings of the 2004 International Conference on New Interfaces for Musical Expression (NIME), Hamamatsu, Japan, 3-5 June 2004, pp. 169-172. National University of Singapore, Singapore.

Wade, N. and Tatler, B. (2005) The Moving Tablet of the Eye: the origins of modern eye movement research. Oxford University Press, USA. 